\title{
Abscissas and Weights for Gaussian Quadratures of High Order
}

\section{P. Davis and P. Rabinowitz}

\begin{abstract}
Weights and abscissas are presented for the Gaussian quadrature rules of order $n=16$, 20, 24, 32, 40, 48. These constants were computed on Standards Automatic Computer by the method described and have passed a number of checks with about 20 places of decimals. Values of the weights and abscissas are also available for $n=64,80$, and 96 .
\end{abstract}

\section{Introduction}

Gaussian quadrature rules have hitherto been computed up to the case $n=16$ with 15 place accuracy [1]. ${ }^{1}$ In the work in the Numerical Analysis Section of the National Bureau of Standards, frequent use has been made of the rule $n=16$ and even of halving and quartering the interval for increased accuracy. For this reason, it is felt that the constants for rules of higher order will prove to be of use in working with electronic digital computers.

For further evidence of the practical utility of high-order rules, the reader may consult Hartree [2], Henrici [3], and Reiz [4]. Exact values of these quantities are also interesting in view of certain unsettled theoretical conjectures that have been made about distribution of the weights and abscissas [7].

\section{Method of Computation}

We deal with the Gaussian quadrature rule of order $n$ on the interval $[-1,1]$ :

$$
\int_{-1}^{+1} f(x) d x=\sum_{k=1}^{n} a_{k n} f\left(x_{k n}\right)
$$

The rule (1) holds exactly whenever $f$ is a polynomial of degree $\leq 2 n-1$. The abscissas $x_{k n}(k=1,2, \ldots, n)$ are the $n$ zeros of the Legendre polynomial of order $n: P_{n}\left(x_{k n}\right)=0$, whereas the weights are given by the expression

$$
a_{k n}=\frac{k_{n+1}}{k_{n}} \frac{-1}{p_{n+1}\left(x_{k n}\right) p_{n}^{\prime}\left(x_{k n}\right)},
$$

where $p_{n} x_{n}=k_{n} x_{n}+\ldots$ are the normalized Legendre polynomials. See, e. g., Szegö [5, p. 47]. Making use of the relationship

$$
\left(1-x^{2}\right) P_{n}^{\prime}(x)=n\left[P_{n-1}(x)-x P_{n}(x)\right],
$$

we are able to derive from (2) the following alternate expression for the weights $a_{k n}$, which is useful for computation:

$$
a_{k n}=\frac{2\left(1-x_{k n}^{2}\right)}{\left[n P_{n-1}\left(x_{k n}\right)\right]^{2}} \quad(k=1,2, \ldots, n) .
$$

\footnotetext{
Figures in brackets indicate the literature references at the end of this paper.
}

To obtain a first approximation to the zeros of the Legendre polynomials, we make use of the following inequality derived by Szegö [6]: Let

Then

$$
x_{k n}=\cos \theta_{k n} \quad(k=1,2, \ldots, n) .
$$

$\frac{j_{k}}{\left[(n+1 / 2)^{2}+c / 4\right]^{1 / 2}}<\theta_{k n}<\frac{j_{k}}{(n+1 / 2)} \quad(k=1,2, \ldots)$,

where $j_{k}(k=1,2, \ldots)$ are the successive zeros of the Bessel function $J_{0}(x)$ and $c=1-(2 / \pi)^{2}$.

The first 150 values of $j_{k}$ may be found in [8]. A preliminary computation of (6) with $n=16$ showed that the value of $\theta_{k n}$ is closer to the left-hand bound, and that five or six decimal places can be secured intially by employing

$$
x_{k n}^{(1)}=\cos \frac{j_{k}}{\left((n+1 / 2)^{2}+c / 4\right)^{1 / 2}}
$$

as a first approximation to $x_{k n}$. The value $x_{k n}^{(1)}$ was successively improved by using the Newton formula

$$
x_{k n}^{(i+1)}=x_{k n}^{(i)}-\frac{P_{n}\left(x_{k n}^{(i)}\right)}{P_{n}^{\prime}\left(x_{k n}^{(i)}\right)} .
$$

The derivative in (8) was computed from (3), whereas the Legendre polynomials themselves were computed from the recursion

$$
\left.\begin{array}{l}
n P_{n}(x)=(2 n-1) x P_{n-1}(x)-(n-1) P_{n-2}(x) \\
P_{0}(x)=1, \quad P_{1}(x)=x
\end{array}\right\}
$$

After the first approximation, the successive approximations were computed in double precision, and a shutoff value of $\epsilon=2^{-74}$ was employed in the iteration (8).

Although the abscissas and the weights are symmetric about $x=0$, all were computed independently. The starting values $x_{k n}\left({ }^{1}\right)$ for two symmetric points were different (cf. (7)). This served as one check of the accuracy of the computation. Additional checks were provided by computing the six quantities given in eq (10), page 37 . 
Abscissas

Weights

$$
\mathrm{n}=2
$$

\section{$0.5773502691 \quad 89625764509$}

$1.0000000000 \quad 0000000000$

$\mathrm{n}=4$

$\begin{array}{ll}0.8611363115 & 94052575224 \\ 0.3399810435 & 84856264803\end{array}$

$\mathrm{n}=8$

0.9602898564 0.7966664774 0.1834346424

97536231684 13626739592 16328985818 95649804939

$\mathrm{n}=16$

0.9894009349 0.9445750230 0.8656312023 0.7554044083 0.6178762444 0.4580167776 0.2816035507 0.0950125098

91649932596 73232576078 87831743880 55003033895 02643748447 57227386342 79258913230 37637440185

$0.3478548451 \quad 3745385737$ $0.6521451548 \quad 6254614262$

0.1012285362

$0.3137066458 \quad 7788728733$

0.36268378337836198296 0.5255324099

$\mathrm{n}=20$

0.9931285991 0.9639719272 0.9122344282 0.83911697 .18 0.7463319064 0.6360536807 0.5108670019 0.3737060887 0.2277858511 0.0765265211
85094924786 77913791268 51325905868 22218823395 60150792614 26515025453 50827098004 15419560673 41645078080 33497333755
0.0176140071 0.0406014298 0.0626720483 0.0832767415 0.1019301198 0.1181945319 0.1316886384 0.1420961093 0.1491729864 0.1527533871

3915211831 0038694133 3410906357 7670474872 1724043503 6151841731 4917662689 1838205132 7260374678 3072585069

\section{$n=24$}

$\begin{array}{ll}0.9951872199 & 97021360180 \\ 0.9747285559 & 71309498198 \\ 0.9382745520 & 02732758524 \\ 0.8864155270 & 04401034213 \\ 0.8200019859 & 73902921954 \\ 0.7401241915 & 78554364244 \\ 0.6480936519 & 36975569252 \\ 0.5454214713 & 88839535658 \\ 0.4337935076 & 26045138487 \\ 0.3150426796 & 96163374387 \\ 0.1911188674 & 73616309159 \\ 0.0640568928 & 62605626085\end{array}$

$0.0123412297 \quad 9998719954$ $0.0285313886 \quad 2893366318$ $0.0442774388 \quad 1741980616$ $0.0592985849 \quad 1543678074$ $0.0733464814 \quad 1108030573$ 0.08619016153195327591 0.09761865210411388827 $0.1074442701 \quad 1596563478$ $0.1155056680 \quad 5372560135$ 0.12167047292780339120 $0.1258374563 \quad 4682829612$ $0.1279381953 \quad 4675215697$
0.9982377097

0.9907262386

0.9772599499

0.9579168192

0.9328128082

0.9020988069

0.8659595032

0.8246122308

0.7783056514

0.7273182551

0.6719566846

0.6125538896

0.5494671250

0.4830758016

0.4137792043

0.3419940908

0.2681521850

0.1926975807

0.1160840706

10559200350 99457006453 83774262663 13791655805 78676533361 68874296728 12259503821 33311663196 26519387695 89927103281 14179548379 67980237953 95128202076 86178712909 71605001525 25758473007 07253681141 01371099716 75255208483 06050821933

$n=48$

0.9987710072 0.9935301722 0.9841245837 0.9705915925 0.9529877031 0.9313866907 0.9058791367 0.8765720202 0.8435882616 0.8070662040 0.7671590325 0.7240341309 0.6778723796 0.6288673967 0.5772247260 0.5231609747 0.4669029047 0.4086864819 0.3487558862 0.2873624873

0.2247637903

0.1612223560 0.0970046992 0.0323801709
52426118601 66350757548 22826857745 46247250461 60430860723 06554333114 15569672822 74247885906 24393530711 29442627083 15740339254 23814654674 32663905212 76513623995 83972703818 22233033678 50958404545 90716729916 92160738160 55455576736 94689061225 68891718056 09462698930 62869362033

$\mathrm{n}=32$

0.0070186100 0.0162743947 0.0253920653 0.0342738629 0.0428358980 0.0509980592 0.0586840934 0.0658222227 0.0723457941 0.0781938957 0.0833119242 0.0876520930 0.0911738786 0.0938443990 0.0956387200 0.0965400885

$n=40$

0.0045212770 0.0104982845 0.0164210583 0.0222458491 0.0279370069 0.0334601952 0.0387821679 0.0438709081 0.0486958076 0.0532278469 0.0574397690 0.0613062424 0.0648040134 0.0679120458 0.0706116473 0.0728865823 0.0747231690 0.0761103619 0.0770398181 0.0775059479

9853319125 3115281361 8190788871 9416695726 8002340109 8254784739 7447201764 8567327199 3507223206 8393682435 9939155136 9292893916 5660103807 1523390382 9128677969 9580405906 5796826420 0062624237 6424796558 7842481126
0.0031533460 0.0073275539 0.0114772345 0.0155793157 0.0196161604 0.0235707608 0.0274265097 0.0311672278 0.0347772225 0.0382413510 0.0415450829 0.0446745608 0.0476166584 0.0503590355 0.0528901894 0.0551995036 0.0572772921 0.0591148396 0.0607044391 0.0620394231 0.0631141922 0.0639242385 0.0644661644 0.0647376968

5230583863 0127626210 7923453949 2294384872 5735552781 3932437914 0835694820 3279808890 


$$
\left.\begin{array}{l}
\sum_{k=1}^{n} a_{k n}=2, \quad \sum_{k=1}^{n} a_{k n} x_{k n}=0, \quad \sum_{k=1}^{n} a_{k n} x_{k n}^{2}=2 / 3 \\
\sum_{k=1}^{n} a_{k n} x_{n}^{3}=0, \quad \sum_{k=1}^{n} a_{k n} x_{n}^{4}=2 / 5, \\
\sum_{k=1}^{n / 2} x_{k n}^{2}=\frac{n(n-1)}{2(2 n-1)}=-\frac{B_{n}}{A_{n}},
\end{array}\right\}
$$

where

$$
P_{n}(x)=A_{n} x^{n}+B_{n} x^{n-1}+\ldots, n \text { even. }
$$

These checks were all met to within 2 units in the 20th decimal place. The first four checks in (10) were carried out on SEAC at the time of the computation, and the last two were made directly from the final tabulation.

In the tables only the abscissas lying between 0 and 1 have been listed.

The authors thank the Hand Computing Unit of the Bureau's Computation Laboratory for its assistance in checking these tables.

\section{References}

[1] A. N. Lowan, N. Davids, and A. Levinson, Table of the zeros of the Legendre polynomials of order $16 \ldots$, Bul. Am. Math. Soc. 48, 739 (1942); reprinted in Tables of functions and of zeros of functions, NBS Applied Mathematics Series 37 (1954).

[2] D. R. Hartree, The evaluation of a diffraction integral, Proc. Cambridge Phil. Soc., p. 567 (1954).

[3] P. Henrici, Application of two methods of numerical analysis to the computation of the reflected radiation of a point source, J. Wash. Acad. Sci. 45, 38 (1955).

[4] A. Reiz, On the numerical solution of certain types of integral equations, Arkiv Mat., Astron. Fysik 29a, 1 (1943).

[5] G. Szegö, Orthogonal polynomials (New York, N. Y., 1939).

[6] G. Szegö, Inequalities for the zeros of the Legendre polynomials and related functions, Trans. Am. Math. Soc. 39, 1 (1936).

[7] G. Szegö (written communication)

[8] British Association Mathematical Tables, vol. VI (Cambridge, 1937).

Washington, July 29, 1955. 